ARTICLE

DOI: $10.1038 / \mathrm{s} 41467-017-00852-6$

OPEN

\title{
A coordination polymer for the site-specific integration of semiconducting sequences into DNA-based materials
}

\author{
Lamia L.G. Al-Mahamad ${ }^{1,2}$, Osama El-Zubir ${ }^{1}$, David G. Smith (10 ${ }^{1,3}$, Benjamin R. Horrocks ${ }^{1}$ \& Andrew Houlton ${ }^{1}$
}

Advances in bottom-up material design have been significantly progressed through DNA-based approaches. However, the routine integration of semiconducting properties, particularly long-range electrical conduction, into the basic topological motif of DNA remains challenging. Here, we demonstrate this with a coordination polymer derived from 6-thioguanosine (6-TG-H), a sulfur-containing analog of a natural nucleoside. The complexation reaction with $\mathrm{Au}(\mathrm{I})$ ions spontaneously assembles luminescent one-dimensional helical chains, characterized as $\left\{\mathrm{Au}^{\mathrm{l}}(\mu-6-\mathrm{TG})\right\}_{n}$, extending many $\mu \mathrm{m}$ in length that are structurally analogous to natural DNA. Uniquely, for such a material, this gold-thiolate can be transformed into a wire-like conducting form by oxidative doping. We also show that this self-assembly reaction is compatible with a 6-TG-modified DNA duplex and provides a straightforward method by which to integrate semiconducting sequences, site-specifically, into the framework of DNA materials, transforming their properties in a fundamental and technologically useful manner.

\footnotetext{
${ }^{1}$ Chemical Nanoscience Labs, School of Chemistry, Newcastle University, Newcastle upon Tyne NE1 7RU, UK. ${ }^{2}$ Department of Chemistry, College of Science, Al-Mustansiriya University, Baghdad, Iraq. ${ }^{3}$ Present address: School of Chemistry, University of Sydney, Sydney, NSW 2006, Australia. Correspondence and requests for materials should be addressed to A.H. (email: andrew.houlton@ncl.ac.uk)
} 


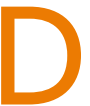

NA, biology's information carrier, is now an established part of preparative chemistry's arsenal for materials synthesis ${ }^{1-3}$. Its robust nature, reliable synthesis, controllable length scale, allied with sequence coding for programmable self-assembly address many of the criteria desired of a materials design toolkit. The ability to incorporate presynthesized inorganic components, particularly nanoparticles, with oligonucleotides provides a logical approach to materials design. This has been demonstrated by the exquisite control over nanoparticle assembly to form DNA-based materials with tunable optical properties ${ }^{4-6}$, reconfigurable superlattice structures ${ }^{7,8}$, and magnetic and catalytic properties ${ }^{9-11}$. However, the integration of semiconducting properties, particularly electrical conduction, into the basic topological framework of duplex DNA remains challenging.

Though studies on the electrical conductivity of duplex DNA have provided conflicting evidence ${ }^{12-14}$, numerous experiments now indicate that it is not an effective solid-state conductor beyond $\mathrm{nm}$ lengths $\mathrm{s}^{15-18}$. Furthermore, effective charge transport appears to require physiological, solution-based, conditions (aqueous buffer etc.) to ensure retention of regular duplex conformation ${ }^{19-22}$. Conditions not well suited to solid-state device applications etc.

Initial indications that, so-called, metallo-DNA or M-DNA ${ }^{23-25}$ offered a solution to long-range electrical conductivity have not been widely adopted; possibly due to conflicting reports ${ }^{26-29}$ and the lack of selectivity over the location of metal-ion incorporation. Modified bases, termed ligandosides, capable of providing specific, non-natural, metal-ion-binding modes have been incorporated into duplex DNA as a means of introducing new properties $^{30-38}$. However, while elegant these approaches are synthetically demanding, and, to date, none provide the extended, delocalized, bonding suited for effective electrical conductivity.

Simple thiopurines are sulfur-containing analogs of natural nucleosides, commonly used in treating a variety of medical conditions. They are readily incorporated into nucleic acids which, in fact, is a feature of their mode of action upon metabolism $^{39}$. Their metal-ion specificity and binding modes are different from the natural derivatives, most notably in their aurophilicity and an ability to form coordination chain polymers via a single-atom-bridge as $\{- \text { Metal- } \mu \mathrm{S}-\}_{n}{ }^{40,41}$. We hypothesized that targeting the synthesis of a gold-based polymer with such a nucleoside variant could provide a material with the required extended bonding motif. Importantly, the aurophilicity of the thiol group would avoid competing reactions with metal-ionbinding sites on the natural nucleosides and allow such a motif to be readily integrated into the framework of duplex DNA structures. Here, we show that the sulfur-containing analog of a natural nucleoside, 6-thioguanosine (6TG-H) reacts with gold(I) ions to spontaneously assemble luminescent helical chains that are structurally analogous to DNA. Uniquely, for such a material, this gold-thiolate coordination polymer can be transformed into a wire-like conducting form. We show that this self-assembly reaction is compatible with a 6-TG-modified DNA duplex and provides a straightforward method by which to integrate semiconductive domains, site-specifically, into DNA materials.

\section{Results}

$\mathrm{Au}(\mathrm{I})$-thioguanosine preparation and characterization. The reaction of equimolar equivalents of $\mathrm{Au}(\mathrm{I})$ ions with the sulfurmodified nucleoside, 6-thioguanosine (6TG-H) in aqueous-based solution at neutral $\mathrm{pH}$ spontaneously forms a luminescent hydrogel (Supplementary Figs. 1, 2). The broad luminescence $\sim 650 \mathrm{~nm}$ is characteristic of the formation of oligomeric goldthiolate species ${ }^{42,} 43$. Further characterization by a range of
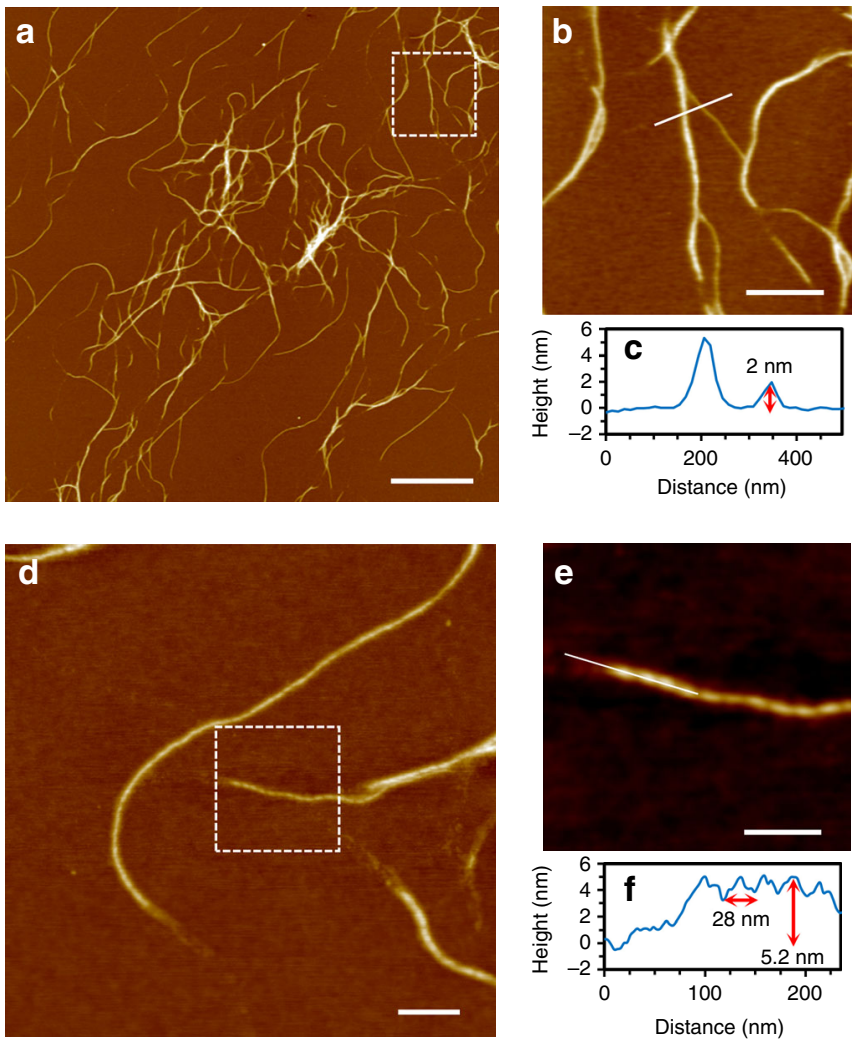

Fig. 1 AFM images of Au-thioguanosine xerogel 1 drop-cast onto a silicon wafer. Large-scan (a) and a zoom area (b) of AFM height images of $\mathbf{1}$. The associated cross-section (c) along the white line in $\mathbf{b}$. AFM height images (d, e) showing the helical structure of individual molecular chains. The associated cross-section (f) along the white line in e. Scale bar is $2 \mu \mathrm{m}$ in image a, $500 \mathrm{~nm}$ in image b, $200 \mathrm{~nm}$ in image d, and $100 \mathrm{~nm}$ in image e

techniques established the reaction to yield a gold(I)-thiolate coordination polymer of general formula $\{-\mathrm{Au}-\mu-6 \mathrm{TG}-\}_{n}$ (Supplementary Discussion and Supplementary Figs. 1-11).

Atomic force microscopy (AFM) imaging (Fig. 1) shows that the dried, xerogel, 1, comprises one-dimensional molecular strands extending many microns in length with the smallest feature heights of ca. $2 \mathrm{~nm}$ (Fig. 1b). These strands entangle to form the necessary three-dimensional network required for gel formation, and can also be seen to intertwine to form larger diameter fibers (Fig. la-c). The AFM data reveals alternating light-dark features along individual fibers consistent with a highly organized helical structure (Fig. 1d, e) ${ }^{42}$. The height of these features indicates that they contain several individual strands and the apparent periodicity seen by AFM arises from intertwining in some relatively ordered supramolecular structure. In support of this, circular dichroism (CD) shows the appearance of markedly more intense bands across the UV-visible range compared to the 6TG-H alone (Supplementary Fig. 4). The longer wavelength bands at ca. 385 and $420 \mathrm{~nm}$ are attributed to metal-ligand charge transfer of the polymer backbone and confirm a helical nature to the main coordination chain.

X-ray diffraction (XRD) data from 1 did not feature sharp Bragg reflections, but instead showed broad peaks indicating that the sample was amorphous (Supplementary Discussion, Supplementary Figs. 5, 6, and Supplementary Table 1). The data were analyzed using a simplified Rietveld method by fitting a regression model comprising a sum of six Gaussian functions. The dominant peaks in Supplementary Fig. 5 are in the range $20<Q<40$ and correspond to distances $d=2 \pi / Q$ of $1.87,2.31$, 
a

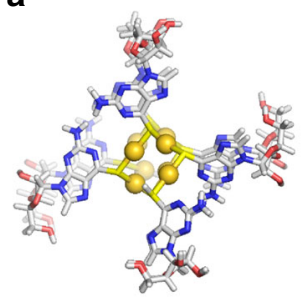

C

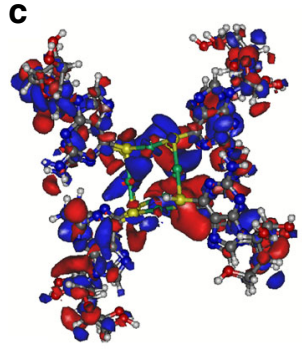

e

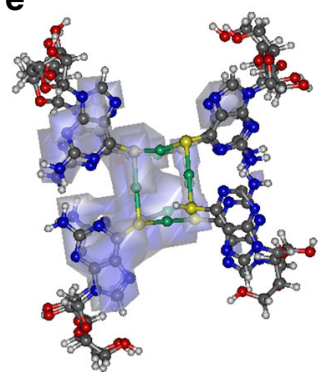

Fig. $2 \mathrm{~A}$ model of the coordination polymer chain of $\{\mathrm{Au}(\mathrm{I}) \text {-thioguanosine }\}_{n}$ viewed along and onto the helical chain axis. $\mathbf{a}, \mathbf{b}$ Single point energy DFT calculations for an oligomer $\left(\mathrm{Au}_{8} 6 \mathrm{TG}_{8}\right)$ using the SBKJC effective core potential and basis set with the B3LYP functional. The helical conformation shown is based on the geometry of comparable Au-thiolates determined by XRD. $\mathbf{c}$ View of the HOMO looking along the axis of the helix; $\mathbf{d}$ The HOMO from a direction normal to the helical axis; e Spin density map for the singly-charged radical cation $\left(\mathrm{Au}_{8} 6 \mathrm{TG}_{8}\right)^{+}$. Looking along the helical axis and $\mathbf{f}$ the spin density map from a direction normal to the helical axis

and $3.18 \AA$. We assign these to $\mathrm{C}-\mathrm{S}, \mathrm{Au}-\mathrm{S}$, and $\mathrm{Au} . . . \mathrm{Au}$ distances, respectively, and the larger $17.6 \AA$ distance can be interpreted as the diameter of the helix.

The available data are all consistent with 1 having a helical structure based on thioguanosine bridging adjacent metal ions analogous to $\mathrm{Au}(\mathrm{I})$-coordination polymers derived, for example, from thiomalate ${ }^{44}$. A molecular model of $\mathbf{1}$ based on this is shown in Fig. 2. The molecular structure has a central polymeric $\mathrm{Au}(\mathrm{I})$ thiolate backbone around which the nucleobase and ribose groups extend. The resulting helical polymer is strikingly similar in size, topology, and surface functionality to duplex DNA.

Electronic structure and electrical properties. Since the asprepared material has both an extended structure and a longwavelength visible luminescence $\left(\lambda_{\max } \sim 650 \mathrm{~nm}\right)$, we naturally considered if it exhibited useful semiconducting properties, analogous to those of conjugated organic polymers ${ }^{45}$. Density functional theory (DFT) calculations of the electronic structure show substantial delocalization of the HOMO, which includes major contributions from the $\{\mathrm{Au}-\mu \mathrm{S}\}_{n}$, backbone as well as minor contributions from the $\pi$-system of the nucleobase. When a single electron is removed from the system and the spin density is computed at the same geometry, this also shows delocalization over both the $\{\mathrm{Au}-\mu \mathrm{S}\}_{n}$ chain and the nucleobase (Fig. 2e, f). The changes in the mean atomic partial charges (Supplementary
Table 1) are largest for the gold-thiolate backbone but with some contribution from the nucleobase. Together these findings suggested that oxidative doping might produce a conductive material and, indeed, this is case. Figure 3 shows data for the electrical characterization of $\mathbf{1}$ using a device constructed by depositing the gel between platinum microband electrodes (MBE) (Fig. 3a-d). After drying, this provided strands of 1 spanning the electrode gap. AFM imaging after treatment with $I_{2}$ vapor showed that no notable structural changes occurred and the molecular strands remained intact (Fig. 3e, f). The chemical effects of this treatment, judged by X-ray photoelectron spectroscopy (XPS), indicated the reaction generally proceed via a redox mechanism with the formation of a polyiodide-doped coordination polymer, $\left(\mathbf{1}_{\mathbf{o x}}\right)$ (Supplementary Fig. 7 and Supplementary Discussion).

Current-voltage curves collected before (1) and after oxidation $\left(\mathbf{1}_{\mathbf{o x}}\right)$ (Fig. 3g) reveal a dramatic increase in conduction upon treatment. Essentially ohmic behavior is observed over the $\pm 2 \mathrm{~V}$ range with an increase of at least two orders of magnitude in conductance upon treatment. The temperature-dependent I-V measurements show Arrhenius behavior characteristic of a thermally activated conduction mechanism with an activation energy of $94 \mathrm{~kJ} \mathrm{~mol}^{-1}$ for $\mathbf{1}_{\mathbf{o x}}$ (Fig. $3 \mathrm{~h}$ ). Alternative oxidants such as $\left(\mathrm{BrC}_{6} \mathrm{H}_{4}\right)_{3} \mathrm{NSbCl}_{6}$ produced similar behavior (Fig. $3 \mathrm{~g}$ ) confirming the conductive nature of the doped coordination polymer $\left(\mathbf{1}_{\mathbf{o x}}\right)$ and that the observed conductivity was not due simply to the presence of the polyiodide species ${ }^{46}$.

The data in Fig. 3 permit several conclusions regarding the nature of the conduction in $\mathbf{1}_{\mathrm{ox}}$. First, it can clearly be seen that the IV curve shows little evidence of conductivity for the undoped material. The differential conductance at zero bias was estimated as $<30 \mathrm{pS}$. The current at high bias, greater than about $1.5 \mathrm{~V}$, shows some increase, but this shape of IV curve, which has almost zero slope at the origin, is not evidence of conductivity in the usual sense. Oxidative doping of $\mathbf{1}$ does produce a material with finite zero bias conductance, whether the oxidant employed is a vapor such as $\mathrm{I}_{2}(7.1+/-0.05 \mathrm{nS})$ or a liquid such as a dry acetonitrile solution containing $\left(\mathrm{BrC}_{6} \mathrm{H}_{4}\right)_{3} \mathrm{NSbCl}_{6}(12.9+/-0.09 \mathrm{nS})$. Photoemission spectra of the doped and undoped material (Supplementary Fig. 7 and Supplementary Discussion) indicate that the positive charge introduced by oxidation resides mainly on $\mathrm{Au}$ and $\mathrm{S}$ atoms. This is supported by DFT calculations, which show large changes in atomic charge for $\mathrm{Au}$ and $\mathrm{S}$, but much smaller changes for atoms of the nucleobase. We conclude that $\mathbf{1}$ shows electronic conductivity upon oxidation and the charge carriers are dominantly associated with the Au-S chains of the structure and not the nucleobase. The IV curves show ohmic behavior, i.e., the slope of the IV curve is the same no matter how small or large the deviation from equilibrium. This behavior is distinct from photoinduced electron transfer experiments in which long-range electron transfer is observed for photon energies sufficient to populate the initial excited state and distinct from electrochemical experiments where the applied potential must exceed the formal potential of the relevant couple to drive electron transfer.

Second, the data in Fig. 3h show that the zero bias conductance increases with temperature in accordance with an equation of Arrhenius form. This is characteristic of a semiconductor or hopping conductor (Supplementary Discussion) and not of a metallic conductor. In principle, the increase in conductance could be explained in terms of thermal excitation across a gap, which would result in an activation energy of $1 / 2 E_{\text {gap }}$. However, the activation energy of about $0.97 \mathrm{eV}$ does not match the data from optical spectroscopy. Fluorescence excitation spectra (Supplementary Fig. 3) show an optical gap of $480 \mathrm{~nm}$, which is about $2.6 \mathrm{eV}$. Instead, the nature of the conductivity in doped $\mathbf{1}_{\mathbf{o x}}$ is more like that of the conjugated polymers than crystalline 

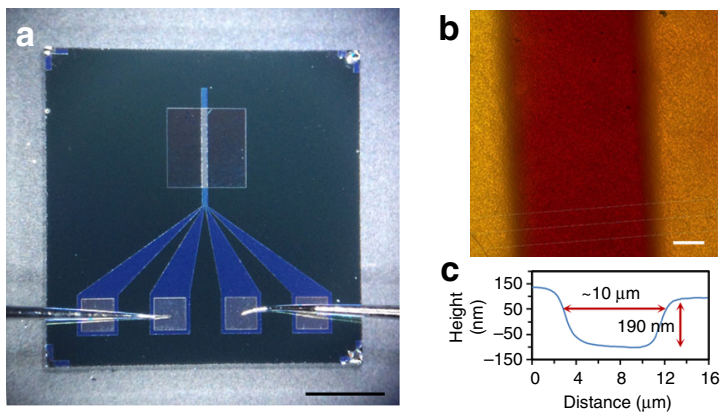

g

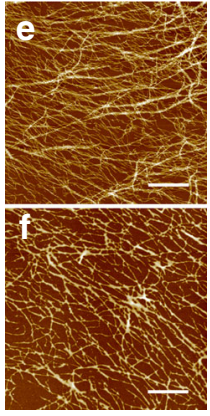

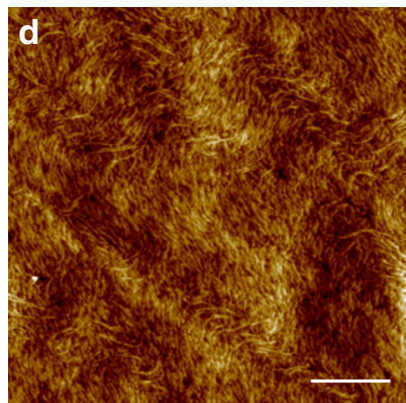

h

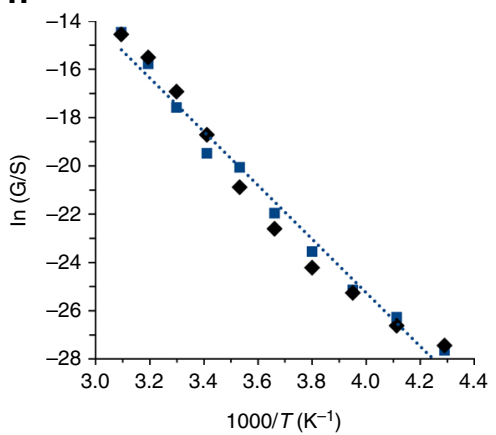

Fig. 3 Electrical characterization of the Au-thioguanosine coordination polymer, 1. a Patterned platinum MBE showing the contact pads at the bottom and the $10 \mu \mathrm{m}$ spaced electrodes above. $\mathbf{b}$, d AFM imaging of the electrode gap region. $\mathbf{c}$ A profile of the gap region indicating the size. AFM images before (e) and after (f) treatment of the xerogel with $\mathrm{I}_{2} \cdot \mathbf{g} \mathrm{I}-\mathrm{V}$ curves of the $\mathbf{1}$ before (blue) and after doping with tris(4-bromophenyl)ammoniumyl hexachloroantimonate (red) and iodine (green). $\mathbf{h}$ An Arrhenius plot for the zero-bias conductance of the $\mathrm{I}_{2}$-doped Au-thioguanosine xerogel. Activation energy $\left(E_{\mathrm{a}}\right)=0.97 \pm 0.02 \mathrm{eV}$ obtained from two independent heating (black)/cooling (blue) cycles. Scale bars are $1 \mathrm{~mm}$ in image $\mathbf{a}$ and $2 \mu \mathrm{m}$ in images $\mathbf{b}$, $\mathbf{d}-\mathbf{f}$

inorganic semiconductors. Typically conjugated polymers are poorly conducting when undoped and have optical gaps of several $\mathrm{eV}$, but conduct via a hopping mechanism when doped. Although bulk samples of conjugated polymers typically show temperaturedependent conductances which follow variable range hopping models ${ }^{47}$, we have observed simple Arrhenius behavior when they are constrained to form as one-dimensional structures ${ }^{48}$. In these systems, and $\mathbf{1}_{\mathbf{o x}}$, the activation energy extracted from the Arrhenius plot is much smaller than the optical gap because it corresponds to thermally assisted tunneling between somewhat localized states rather than carrier generation by excitation across the gap as in crystalline inorganic semiconductors. Finally, it is worth noting that we observed essentially identical Arrhenius plots for both heating and cooling cycles and the doped polymer $\mathbf{1}_{\mathbf{o x}}$ is stable up to at least $50^{\circ} \mathrm{C}$.

Site-specific incorporation into DNA duplex. Next, we sought to establish the compatibility of this self-assembly chemistry with DNA oligonucleotides for the site-specific incorporation of this semiconducting $\mathrm{Au}^{\mathrm{I}}$-thiolate motif into DNA sequences. To this end we designed an 18-mer oligonucleotide with the sequence 5'-SSSSACGCGAATTCGCGT-3' (where S=6-deoxythioguano sine, dTG-H), which contains a self-complementary 14-mer region. This was annealed to form the corresponding duplex $\left\{\right.$ OligoS $\left._{4}\right\}$ with $5^{\prime}$ overhangs with four consecutive 6-dTG-H groups. CD spectroscopy confirmed the formation of B-form duplex with negative-positive bands seen at $\sim 250$ and $\sim 284 \mathrm{~nm}$ (Supplementary Fig. 9). Longer wavelength bands were also observed at $\sim 330 \mathrm{~nm}$ (negative) and $\sim 354 \mathrm{~nm}$ (positive) which are due to transitions associated with the 6-dTG-H sequence. Reactions of $\left\{\mathrm{OligoS}_{4}\right\}$ with $\mathrm{Au}(\mathrm{I})$ ions exhibited an orange luminescence similar to $1 \mathrm{ca} .520 \mathrm{~nm}$ indicating the spontaneous formation of oligomeric $\mathrm{Au}(\mathrm{I})$-thiolate species (Supplementary
Fig. 10). The blue-shift in this emission compared to 1 can be rationalized by the short length of the Au-thiolate region in the Au- $\left\{\right.$ OligoS $\left._{4}\right\}$. The self-assembly is also indicated by CD with a red-shift in the signal associated with the 6-dTG sequence and a transformation of the duplex region to an A-form conformation (Supplementary Fig. 9).

The successful integration and compatibility of the coordination polymer motif with the DNA duplex was revealed by AFM. This showed one-dimensional Au- $\left.\mathbf{A} \mathbf{O l i g o S}_{4}\right\}$ structures extending up to several microns in length indicating coaxial alignment of the native duplex and the gold-thiolate sequences (Fig. 4a, b). The observed one-dimensional structures suggest that branching while, in principle, possible is not dominant as this would form a cross-linked network. Branched structures may in principle arise from either two or more strands that intertwine for part of their length or by joining three duplexes via $\mathrm{Au}-\mathrm{S}$ links. The former case can be distinguished in AFM by the diameter change at the branch point. Examination of the data (Fig. 4a and Supplementary Fig. 11a-f) shows that this is the typical case. Similar large features are not present in samples of $\left\{\mathbf{O l i g o S}_{\mathbf{4}}\right\}$ alone; these samples generally contain much shorter (100 $\mathrm{s} \mathrm{nm})$ fibers that can aggregate into bundles (Supplementary Fig. 12e-h).

The mechanism by which the $\mathbf{A u}-\left\{\mathbf{O l i g o S}_{4}\right\}$ strands are produced is driven by coordinate bond formation involving the 6-thioguanosine terminal regions. While other donor atom sites (i.e., N, O) are present in the nucleoside bases, the high affinity of $\mathrm{Au}(\mathrm{I})$ for the mutant thiol-functionality provides the sequencespecificity for the coordination motif. The resulting hybrid material comprise alternating sections of native double helices linked by semiconducting oligo-Au(I)-TG regions (Fig. 4). As individual $\left\{\right.$ OligoS $\left._{4}\right\}$ are only ca. $6 \mathrm{~nm}$ long extensive concatenation involving hundreds and thousands of individual DNA duplexes occurs. In fact, fluorescence microscopy indicates that these assemblies can be macroscopic, extending in length to 

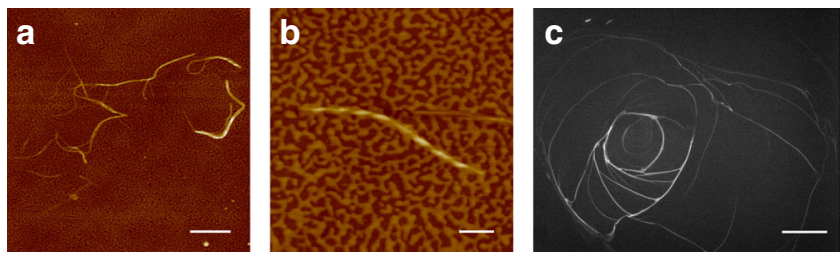

d

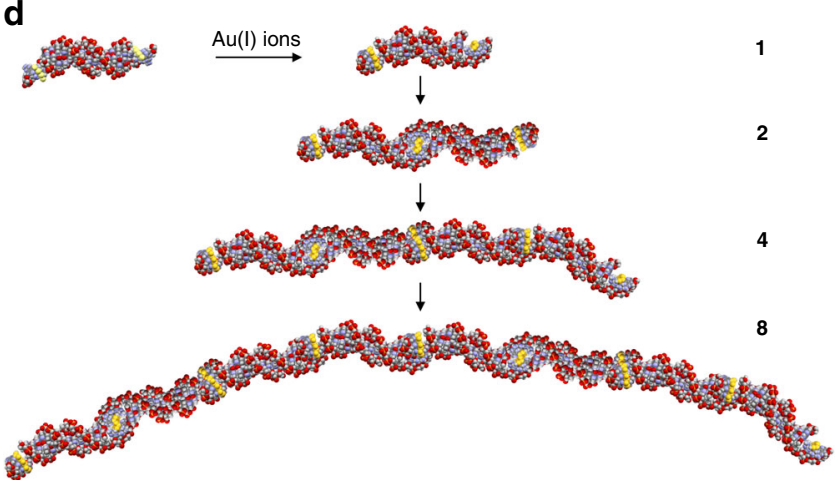

Fig. 4 Au-thiolate-DNA concatemers. AFM height image of Au-[Oligos $\mathbf{4}]$ revealing the extended, micron, lengths of strands formed (a) and the helical nature of an individual structure (b). c Fluorescence image of $\mathbf{A u}$-[Oligos $\mathbf{4}]$ revealing the macroscopic fibers. $\mathbf{d}$ Schematic illustration of the formation of DNA-semiconductor concatemer sequence strands by metal-ion-driven self-assembly. Yellow indicates the semiconducting Au-thiolate region, red $=$ Oxygen, purple $=$ Nitrogen, gray $=$ Carbon. Scale bar is $500 \mathrm{~nm}$ in image $\mathbf{a}, 100 \mathrm{~nm}$ in image $\mathbf{b}$, and $1 \mathrm{~mm}$ in image $\mathbf{c}$

several mm (Fig. 4c). Closer inspection of individual structures of $\mathbf{A u}-\left\{\right.$ OligoS $\left._{4}\right\}$ reveals, again, an alternating pattern of light-dark features along the length indicating helicity in the hybrid strands (Fig. 4b). The periodicity is consistent with the highly organized underlying structure expected from the self-assembly process outlined in Fig. 4d.

\section{Discussion}

In summary, we have prepared a luminescent one-dimensional coordination polymer based on the self-assembly of $\mathrm{Au}^{\mathrm{I}}$ ions with the sulfur-modified nucleoside, 6-thioguanosine. The material's molecular structure is highly analogous to duplex DNA and this polymeric gold-thiolate, uniquely to our knowledge, exhibits electrical conduction upon oxidative doping. This semiconducting motif can be readily incorporated, co-axially and sequencespecifically, into the framework of DNA structures due to the metal-ion binding that assembles the coordination chain being specific for the mutant nucleoside. With the increasingly important role of DNA in bottom-up nanoscience and material synthesis ${ }^{2}, 4-6,8,9,11$, routes to integrate technologically useful physico-chemical properties with DNA are highly sought. This has typically been achieved with pre-synthesized components such as nanoparticles, or by deposition of metals and inorganics on the DNA ${ }^{49}$. Our work advances the field of DNA materials toward further complexity by allowing the incorporation of semiconducting properties via an alternative method and makes possible new types of construction protocols, compositional architectures, and material combinations.

\section{Methods}

Materials. Reagents were obtained from Sigma-Aldrich. NANOpure ${ }^{\circledR}$ deionized water $\left(18 \mathrm{M} \Omega \mathrm{cm}\right.$ resistivity) was obtained from a NANOpure ${ }^{\circledR}$ DIamond ${ }^{\mathrm{TM}}$ Life Science ultrapure water system equipped with a DIamond ${ }^{\text {Tm }}$ RO Reverse Osmosis System (Barnstead International). The thio-modified 18-mer oligonucleotide, $5^{\prime}$-SSSSACGCGAATTCGCGT- $3^{\prime}$ where $\mathrm{S}=6$-thiodeoxyguanosine, containing a 14-mer self-complementary region was obtained from ATDBio (Southampton, UK). Oligonucleotides were made up in $10 \mathrm{mM}$ HEPES buffer containing $50 \mathrm{mM}$ $\mathrm{NaCl}$ and adjusted to $\mathrm{pH}=7$.

Preparation of 1. Sample-spanning gels of 1 were prepared as follows. An aqueous suspension of 6-thioguanosine (6TG-H, $10 \mathrm{mg}$ ) was sonicated for $30 \mathrm{~s}$ to achieve a fine dispersion and to this was added an equimolar equivalent of $\mathrm{Au}(\mathrm{I})$ ions, formed by reduction of $\mathrm{HAuCl}_{4}$ with two equivalents of thiodiglycol, $\mathrm{HO}\left(\mathrm{CH}_{2}\right)_{2} \mathrm{~S}$ $\left(\mathrm{CH}_{2}\right) \mathrm{OH}$ in a final volume of $600 \mu \mathrm{l}$. Upon addition of the $\mathrm{Au}(\mathrm{I})$ ion a yelloworange gel is formed which is stable to inversion in a vial (Supplementary Fig. 1). Alternatively, reactions could be performed in a $(4: 1) \mathrm{H}_{2} \mathrm{O} / \mathrm{MeOH}$ mixture with dissolution of the $6 \mathrm{TG}-\mathrm{H}$ by adding $\mathrm{NaOH}$. The remainder of the reaction was performed as above. These gels contain chloride ions from the gold salt and the thiodiglycol-derived oxidation product as the corresponding sulfone, $\mathrm{HO}$ $\left(\mathrm{CH}_{2}\right)_{2} \mathrm{~S}=\mathrm{O}\left(\mathrm{CH}_{2}\right) \mathrm{OH}$, as indicated by XPS (see below) and confirmed by singlecrystal X-ray crystallography in the latter case.

FTIR spectroscopy. FTIR spectra (in the range $800-4000 \mathrm{~cm}^{-1}$ ) were recorded in transmission mode on a Varian 800 Scimitar Series. Samples of $1(\sim 2 \mu \mathrm{l})$ was deposited on a clean $\mathrm{p}$-Si $(100)$ chip $\left(1 \mathrm{~cm}^{2}\right)$ and dried in air for $1 \mathrm{~h}$ prior to analysis (Supplementary Fig. 3).

Electrospray mass spectrometry. ES-MS spectra were recorded with a Waters Micromass LCT Premier mass spectrometer. Sample requirements for this analysis necessitated dissolution of $\mathbf{1}$ in methanol:water (50:50) with gentle heating.

Fluorescence spectroscopy. Emission and excitation spectra were recorded on a SPEX Fluoromax spectrofluorimeter. Gels of 1 are luminescent, exhibiting an emission band at $\sim 650 \mathrm{~nm}\left(\lambda_{\mathrm{ex}}=480 \mathrm{~nm}\right)$ (Supplementary Fig. 2).

Circular dichroism. CD spectra were recorded on a Jasco J-810 spectrometer. $\mathrm{CD}$ spectra of aqueous 6-thioguanosine $\left(1 \mathrm{mg} \mathrm{ml}^{-1}\right)$ were recorded in a quartz cell with pathlength $0.2 \mathrm{~mm}$. CD spectra of 1 as a gel (prepared with $10 \mathrm{mg} / 1.8 \mathrm{ml}$ of 6-thioguanosine) were recorded in a quartz cuvette of pathlength $0.1 \mathrm{~mm}$. In order to facilitate direct, quantitative comparison of the two samples, the ellipticity for the 6-thioguanosine (Supplementary Fig. 4a) has been multiplied by a factor $10 / 3.6$ to account for the difference in concentration and pathlength.

Atomic force microscopy. AFM data was acquired using a Multimode 8 atomic force microscope with a NanoscopeV controller (Bruker) and a "E" scanner. Nanoscope software version 9.1 was used to control the microscope. The system was operated in ScanAsyst in Air mode as a peak force tapping mode at ultra-low forces minimize damage to the samples. For reducing vibrational noise, an isolation table/acoustic enclosure was used (Veeco Inc., Metrology Group). Silicon tips on silicon nitride cantilevers (ScanAsyst, Bruker) were used for imaging. The nominal tip radius was approximately $2 \mathrm{~nm}$, resonant frequency $150 \mathrm{kHz}$, and spring constant $k \sim 0.7 \mathrm{~nm}^{-1}$. The AFM data were analyzed with NanoScope Analysis 1.5 software (Bruker). The sample was prepared by adding $2 \mu \mathrm{l}$ of 1 onto a clean silicon wafer and drying in air.

Powder XRD. XRD data were acquired using of a PANalytical X'Pert Pro diffractometer (PANalytical) using $\mathrm{Cu} \mathrm{K \alpha}$ radiation source $(\lambda=1.540 \AA)$. Samples of 1 were dried onto glass slides.

X-ray photoelectron spectroscopy. XPS spectra were acquired using a Thermo Scientific K-Alpha X-ray photoelectron spectrometer (Thermo Electron Corp., Eas Grinstead, UK), equipped with an Al Ka X-ray source (1486.6 eV). A take-off angle of $90^{\circ}$ was used during data acquisition, and a charge neutralization gun used to compensate for surface charging. The CasaXPs software (Casa, http://www.casaxps. com, UK) was used to analyze the XPS spectra. All spectra were corrected to hydrocarbon $\mathrm{C} 1 \mathrm{~s}$ peak at $285 \mathrm{eV}$ as reference. Wide scans spectra were recorded at a pass energy of 150 and $1 \mathrm{eV} / \mathrm{step}$, while narrow scans spectra were recorded at a pass energy of 50 and $0.1 \mathrm{eV} / \mathrm{step}$.

Electrical measurements. All of the electrical measurements were carried out under dry nitrogen without light illumination. Current-voltage measurements at different temperatures were measured on the probe station (Cascade Microtech with a B1500A parameter analyzer, Agilent) using a thermal chuck system (Model ETC-200L, ESPEC, Japan). Platinum MBEs (Smart Microsystems Pt MB-4000, Windsor Scientific Ltd., Slough, UK) were used to fabricate electronic devices for electrical characterization of Au-thioguanosine xerogel. The MBEs were made on $\mathrm{Si} / \mathrm{SiO}_{2}$ substrates. Four independent platinum electrodes were patterned on the top of the $\mathrm{SiO}_{2}$ layer. The height of the electrodes is $200 \mathrm{~nm}$ and their width is $10 \mu \mathrm{m}$ with $10 \mu \mathrm{m}$ spaces between them. The surfaces of the MBEs were electrically insulated except for a $2 \times 2 \mathrm{~mm}^{2}$ area for depositing the gel, as shown in Fig. $3 \mathrm{a}$. Devices were fabricated by depositing the gel onto the platinum MBEs. The platinum MBEs were washed with ethanol and dried with nitrogen gas. The clean 
platinum electrodes were analyzed on a probe station and reference current/voltage curves were recorded, which showed the background currents to be less than 100 $\mathrm{fA}$ at $2 \mathrm{~V}$. For the device a drop $(\sim 1 \mu \mathrm{l})$ of Au-thioguanosine gel $\mathbf{1}$ was cast onto the uninsulated area on the MBE. The device was left to dry for a week in Schlenk flask, which was maintained on a vacuum line $(\sim 750 \mathrm{mbar})$ at a temperature of $45^{\circ} \mathrm{C}$. The gel droplet dried to produce a film of the Au-thioguanosine fibers across the Pt-electrodes, as shown in Fig. 3b, d. The electrodes were connected to the probe station and current/voltage curves were collected. For doping, the xerogel $\mathbf{1}$ was exposed to iodine or tris(4-bromophenyl)ammoniumylhexachloroantimonate, $\left(\mathrm{BrC}_{6} \mathrm{H}_{4}\right)_{3} \mathrm{NSbCl}_{6}$. In the former case, $\mathbf{1}$ on platinum MBEs was exposed to iodine vapor for $1 \mathrm{~h}$ by heating $0.1 \mathrm{~g}$ of iodine to $45^{\circ} \mathrm{C}$ in a $50 \mathrm{ml}$ round bottom glass flask. For the latter 1 on MBEs was treated with a drop $(\sim 1 \mu \mathrm{l})$ of $0.1 \mathrm{~mol} \mathrm{dm}^{-3}$ of $\left(\mathrm{BrC}_{6} \mathrm{H}_{4}\right)_{3} \mathrm{NSbCl}_{6}$ in anhydrous acetonitrile. The gel was left to dry for $30 \mathrm{~min}$ before the current/voltage curves were collected. Control experiments where iodine or $\left(\mathrm{BrC}_{6} \mathrm{H}_{4}\right)_{3} \mathrm{NSbCl}_{6}$ was applied to MBEs in the absence of $\mathbf{1}$ showed negligible background currents. In other control experiments DNA was deposited on MBEs and subsequently treated in a similar manner to the samples for $\mathbf{1}$ (Supplementary Fig. 8).

Reaction of 6-TG-modified oligonucleotides with $\mathbf{A u ( I )}$ ions. An 18-mer oligonucleotide, $\mathrm{OligoS}_{4}$, containing a 14-mer self-complementary sequence [5'-SSSSACGCGAATTCGCGT-3' where $\mathrm{S}=6$-thiodeoxyguanosine], with a tetrameric $5^{\prime}$-6-thiodeoxyguanosine overhang region was annealed by heating to $70^{\circ} \mathrm{C}$ and cooled to room temperature. Duplex formation was confirmed by $\mathrm{CD}$, which indicated the expected negative-positive bands seen at $\sim 250$ and $\sim 284 \mathrm{~nm}$ for B-form DNA ${ }^{50}$. Longer wavelength bands were also observed at $\sim 330 \mathrm{~nm}$ (negative) and $\sim 354 \mathrm{~nm}$ (positive) which are due to transitions associated with the 6-thioguanosine section (Supplementary Fig. 9) ${ }^{51}$.

A typical reaction of the $\left[\mathrm{OligoS}_{4}\right]$-duplex and $\mathrm{Au}(\mathrm{I})$ ions was as follows: to $20 \mu \mathrm{l}$ of a $1 \mathrm{mM}$ [Oligos 4 ] solution was added an $\mathrm{Au}(\mathrm{I})$ solution (prepared by reduction of $\mathrm{HAuCl}_{4} .3 \mathrm{H}_{2} \mathrm{O}$ using two equivalents of thiodiglycol) to give an gold: sulfur ratio of 1:1. This mixture exhibited an orange luminesce (Supplementary Fig. $10 \mathrm{a}-\mathrm{c}$ ) and a very broad emission peak at $\sim 520 \mathrm{~nm}$ (Supplementary Fig. 10d). The CD spectra of Au-[OligoS 4 ] shows bands at $\sim 275 \mathrm{~nm}$ (positive), $\sim 340 \mathrm{~nm}$ (negative), and $375 \mathrm{~nm}$ (positive). These changes indicate a transformation to a more A-like conformation of the native duplex region as well as changes to the thioguanosine section ${ }^{50}$.

Fluorescence microscopy. Fluorescence images were obtained using a Zeiss Epifluorescence Microscope equipped with a Hg lamp and a bandpass filter for the excitation of $300-400 \mathrm{~nm}$. A long-pass filter with a cut off at $420 \mathrm{~nm}$ was used for detection and the image was collected with a monochrome CCD camera.

Data availability. All data are available from the authors upon reasonable request.

Received: 15 February 2017 Accepted: 31 July 2017

Published online: 28 September 2017

\section{References}

1. Meng, W. J. et al. An autonomous molecular assembler for programmable chemical synthesis. Nat. Chem. 8, 542-548 (2016).

2. Jones, M. R., Seeman, N. C. \& Mirkin, C. A. Programmable materials and the nature of the DNA bond. Science 347, 1260901 (2015).

3. Aldaye, F. A., Palmer, A. L. \& Sleiman, H. F. Assembling materials with DNA as the guide. Science 321, 1795-1799 (2008).

4. Sun, D. \& Gang, O. Binary heterogenous superlattices assembled from quantum dots and gold nanoparticles with DNA. J. Am. Chem. Soc. 133, 5252-5254 (2011).

5. Young, K. L. et al. Using DNA to design plasmonic metamaterials with tunable optical properties. Adv. Mater. 26, 653-659 (2014).

6. Park, D. J. et al. Plasmonic photonic crystals realized through DNAprogrammable assembly. Proc. Natl Acad. Sci. USA 112, 977-981 (2015).

7. Kim, Y., Macfarlane, R. J. \& Mirkin, C. A. Dynamically interchangeable nanoparticle superlattices through the use of nucleic acid-based allosteric effectors. J. Am. Chem. Soc. 135, 10342-10345 (2013).

8. Macfarlane, R. J., Jones, M. R., Lee, B., Auyeung, E. \& Mirkin, C. A. Topotactic interconversion of nanoparticle superlattices. Science 341, 1222-1225 (2013).

9. Zhang, C. et al. A general approach to DNA-programmable atom equivalents. Nat. Mater. 12, 741-746 (2013)

10. Radha, B. et al. Reconstitutable nanoparticle superlattices. Nano Lett. 14, 2162-2167 (2014).

11. Auyeung, E. et al. Controlling structure and porosity in catalytic nanoparticle superlattices with DNA. J. Am. Chem. Soc. 137, 1658-1662 (2015).

12. Fink, H. W. \& Schonenberger, C. Electrical conduction through DNA molecules. Nature 398, 407-410 (1999).
13. Dekker, C. \& Ratner, M. A. Electronic properties of DNA. Phys. World 14, 29-33 (2001)

14. Endres, R. G., Cox, D. L. \& Singh, R. R. P. The quest for high-conductance DNA. Rev. Mod. Phys. 76, 195 (2004).

15. Storm, A. J., van Noort, J., de Vries, S. \& Dekker, C. Insulating behavior for DNA molecules between nanoelectrodes at the $100 \mathrm{~nm}$ length scale. Appl. Phys. Lett. 79, 3881-3883 (2001).

16. Gomez-Navarro, C. et al. Contactless experiments on individual DNA molecules show no evidence for molecular wire behavior. Proc. Natl Aacd. Sci. USA 99, 8484-8487 (2002).

17. Bockrath, M. et al. Scanned conductance microscopy of carbon nanotubes and lambda-DNA. Nano Lett. 2, 187-190 (2002).

18. Sonmezoglu, S., Sonmezoglu, O. A., Cankaya, G., Yildirim, A. \& Serin, N. Electrical characteristics of DNA-based metal-insulator-semiconductor structures. J. Appl. Phys. 107, 124518 (2010).

19. Guo, X. F., Gorodetsky, A. A., Hone, J., Barton, J. K. \& Nuckolls, C. Conductivity of a single DNA duplex bridging a carbon nanotube gap. Nat. Nanotechnol. 3, 163-167 (2008).

20. Slinker, J. D., Muren, N. B., Renfrew, S. E. \& Barton, J. K. DNA charge transport over $34 \mathrm{~nm}$. Nat. Chem. 3, 228-233 (2011).

21. Sontz, P. A., Muren, N. B. \& Barton, J. K. DNA charge transport for sensing and signaling. Acc. Chem. Res. 45, 1792-1800 (2012).

22. O'Brien, E. et al. The $4 \mathrm{Fe} 4 \mathrm{~S}$ cluster of human DNA primase functions as a redox switch using DNA charge transport. Science 355, 813 (2017).

23. Aich, P. et al. M-DNA: a complex between divalent metal ions and DNA which behaves as a molecular wire. J. Mol. Biol. 294, 477-485 (1999).

24. Rakitin, A. et al. Metallic conduction through engineered DNA: DNA nanoelectronic building blocks. Phys. Rev. Lett. 86, 3670-3673 (2001).

25. Whittell, G. R. \& Manners, I. Metallopolymers: new multifunctional materials. Adv. Mater. 19, 3439-3468 (2007).

26. Moreno-Herrero, F. et al. Topographic characterization and electrostatic response of M-DNA studied by atomic force microscopy. Nanotechnology 14, 128-133 (2003).

27. Liu, B., Bard, A. J., Li, C.-Z. \& Kraatz, H.-B. Scanning electrochemical microscopy. 51. Studies of self-assembled monolayers of DNA in the absence and presence of metal ions. J. Phys. Chem. B 109, 5193-5198 (2005).

28. Mizoguchi, K., Tanaka, S., Ogawa, T., Shiobara, N. \& Sakamoto, H. Magnetic study of the electronic states of B-DNA and M-DNA doped with metal ions. Phys. Rev. B 72, 033106 (2005)

29. Spring, B. Q. \& Clegg, R. M. Fluorescence measurements of duplex DNA oligomers under conditions conducive for forming M-DNA (a metal-DNA complex). J. Phys. Chem. B 111, 10040-10052 (2007).

30. Clever, G. H., Kaul, C. \& Carell, T. DNA-metal base pairs. Angew. Chem. Int Ed. 46, 6226-6236 (2007).

31. Clever, G. H. \& Shionoya, M. Metal-base pairing in DNA. Coord. Chem. Rev. 254, 2391-2402 (2010)

32. Takezawa, Y. \& Shionoya, M. Metal-mediated DNA base pairing: alternatives to hydrogen-bonded Watson-Crick base pairs. Acc. Chem. Res. 45, 2066-2076 (2012).

33. Atwell, S., Meggers, E., Spraggon, G. \& Schultz, P. G. Structure of a coppermediated base pair in DNA. J. Am. Chem. Soc. 123, 12364-12367 (2001).

34. Wagenknecht, H. A. Metal-mediated DNA base pairing and metal arrays in artificial DNA: towards new nanodevices. Angew. Chem. Int. Ed. 42, 3204-3206 (2003).

35. Sinha, I., Guerra, C. F. \& Mueller, J. A highly stabilizing silver(I)-mediated base pair in parallel-stranded DNA. Angew. Chem. Int. Ed. 54, 3603-3606 (2015).

36. Johannsen, S., Megger, N., Bohme, D., Sigel, R. K. O. \& Muller, J. Solution structure of a DNA double helix with consecutive metal-mediated base pairs. Nat. Chem. 2, 229-234 (2010).

37. Scharf, P. \& Muller, J. Nucleic acids with metal-mediated base pairs and their applications. ChemPlusChem 78, 20-34 (2013).

38. Santamaría-Díaz, N., Méndez-Arriaga, J. M., Salas, J. M. \& Galindo, M. A. Highly stable double-stranded DNA containing sequential silver(I)-mediated 7deazaadenine/thymine Watson-Crick base pairs. Angew. Chem. Int. Ed. 55, 6170-6174 (2016).

39. Aarbakke, J., Janka-Schaub, G. \& Elion, G. B. Thiopurine biology and pharmacology. Trends Pharmacol. Sci. 18, 3-7 (1997).

40. Olea, D. et al. From coordination polymer macrocrystals to nanometric individual chains. Adv. Mater. 17, 1761-1765 (2005).

41. Amo-Ochoa, P. et al. Coordination chemistry of 6-thioguanine derivatives with cobalt: toward formation of electrical conductive one-dimensional coordination polymers. Inorg. Chem. 52, 5290-5299 (2013).

42. Lavenn, C. et al. A luminescent double helical gold(I)-thiophenolate coordination polymer obtained by hydrothermal synthesis or by thermal solid-state amorphous-to-crystalline isomerization. J. Mater. Chem. C 3, 4115-4125 (2015).

43. Odriozola, I., Loinaz, I., Pomposo, J. A. \& Grande, H. J. Gold-glutoathione supramolecular hydrogels. J. Mater. Chem. 17, 4843-4845 (2007). 
44. Bau, R. Crystal structure of the antiarthritic drug gold thiomalate (myochrysine): a double-helical geometry in the solid state. J. Am. Chem. Soc. 120, 9380-9381 (1998).

45. Skotheim, T. A. \& Reynolds, J. Handbook of Conducting Polymers, 2 Volume Set. 1680 (CRC Press, 2007).

46. Hardy, L. C. \& Shiver, D. F. Poly (ethylene oxide)-sodium polyiodide conductors: characterization, electrical conductivity, and photoresponse. J. Am. Chem. Soc. 108, 2887-2893 (1986).

47. Joo, J. et al. Physical characterization of electrochemically and chemically synthesized polypyrroles. Macromolecules 33, 5131-5136 (2000).

48. Hassanien, R. et al. Preparation and characterization of conductive and photoluminescent DNA-templated polyindole nanowires. ACS Nano 4 2149-2159 (2010).

49. Houlton, A. \& Watson, S. M. D. DNA-based nanowires. Towards bottom-up nanoscale electornics. Ann. Rep. Prog. Chem. A 107, 21-42 (2011).

50. Nordén, B., Rodger, A. \& Dafforn, T. Linear Dichroism and Circular Dichroism: A Textbook on Polarized-Light Spectroscopy (RSC, 2010).

51. Repges, R., Beuck, C., Weinhold, E., Raabe, G. \& Fleischhauer, J. 6-Thioguanosine in DNA as CD-spectroscopic probe to study local structural changes upon protein binding. Chirality 20, 978-984 (2008).

\section{Acknowledgements}

We gratefully acknowledge funding support from the EPSRC (EP/K018051/1). L.L.G.A.-M. acknowledges the Iraqi Ministry of Higher Education and Scientific Research for a scholarship.

\section{Author contributions}

L.L.G.A.-M., O.E.-Z., D.G.S., B.R.H., A.H. performed experiments and collected and analyzed data. L.L.G.A.-M. and O.E.-Z. contributed equally to the experimental work.
B.R.H. and A.H. designed experiments. O.E.-Z., B.R.H., and A.H. wrote the manuscript. A.H. designed the project.

\section{Additional information}

Supplementary Information accompanies this paper at doi:10.1038/s41467-017-00852-6.

Competing interests: The authors declare no competing financial interests.

Reprints and permission information is available online at http://npg.nature.com/ reprintsandpermissions/

Publisher's note: Springer Nature remains neutral with regard to jurisdictional claims in published maps and institutional affiliations.

\begin{abstract}
(c) (i) Open Access This article is licensed under a Creative Commons Attribution 4.0 International License, which permits use, sharing, adaptation, distribution and reproduction in any medium or format, as long as you give appropriate credit to the original author(s) and the source, provide a link to the Creative Commons license, and indicate if changes were made. The images or other third party material in this article are included in the article's Creative Commons license, unless indicated otherwise in a credit line to the material. If material is not included in the article's Creative Commons license and your intended use is not permitted by statutory regulation or exceeds the permitted use, you will need to obtain permission directly from the copyright holder. To view a copy of this license, visit http://creativecommons.org/ licenses/by/4.0/.
\end{abstract}

(C) The Author(s) 2017 